\title{
EIGENVALUES OF SCHRÖDINGER OPERATORS NEAR THRESHOLDS: TWO TERM APPROXIMATION
}

\author{
YURIY GOLOVATY
}

Abstract. We consider one dimensional Schrödinger operators

$$
H_{\lambda}=-\frac{d^{2}}{d x^{2}}+U+\lambda V_{\lambda}
$$

with nonlinear dependence on the parameter $\lambda$ and study the small $\lambda$ behavior of eigenvalues. Potentials $U$ and $V_{\lambda}$ are real-valued bounded functions of compact support. Under some assumptions on $U$ and $V_{\lambda}$, we prove the existence of a negative eigenvalue that is absorbed at the bottom of the continuous spectrum as $\lambda \rightarrow 0$. We also construct two-term asymptotic formulas for the threshold eigenvalues.

\section{INTRODUCTION}

About forty years ago, Simon and Klaus [1-4] started studying the low energy behavior of the so-called weakly coupled Hamiltonians $-\Delta+\lambda V$. The considerable interest has been in the study of negative-energy bound states and their small $\lambda$ behavior, as well as in the study of the absorption of the eigenvalues by the continuous spectrum. The main results here have been concerned with Schrödinger operators in one and two dimensions, because in three dimensions the weakly coupled Hamiltonians have no bound state if $\lambda$ is small enough, i.e., if potential $\lambda V$ is a sufficiently shallow well. For the case of 1D Hamiltonians $\mathcal{H}_{\lambda}=-\frac{d^{2}}{d x^{2}}+\lambda V$, a suitable short-range potential $V$ can produce a bound state for all small $\lambda$. Assuming that $V$ is different from zero and $\int_{\mathbb{R}}\left(1+|x|^{2}\right)|V(x)| d x<$ $\infty$, Simon [1] proved that the operator $\mathcal{H}_{\lambda}$ has a negative-energy bound state $e_{\lambda}$ for all small positive $\lambda$ if and only if $\int_{\mathbb{R}} V(x) d x \leq 0$. If $\mathcal{H}_{\lambda}$ does have an eigenvalue, then it is unique and simple, and obeys

$$
\sqrt{-e_{\lambda}}=-\frac{\lambda}{2} \int_{\mathbb{R}} V(x) d x-\frac{\lambda^{2}}{4} \iint_{\mathbb{R}^{2}} V(x)|x-y| V(y) d x d y+o\left(\lambda^{2}\right)
$$

as $\lambda \rightarrow 0$. This asymptotic formula is due to Abarbanel, Callan and Goldberger, but it was not published by them; (1) was firstly announced by Simon [1]. The eigenvalue $e_{\lambda}$ approaches zero as $\lambda$ goes to zero and it is absorbed in the limit at the bottom of the continuous spectrum $[0,+\infty)$. Then we say that $\lambda=0$ is a coupling constant threshold for $\mathcal{H}_{\lambda}$. Klaus [2] has extended this result to the class of potentials $V$ obeying the condition $\int_{\mathbb{R}}(1+|x|)|V(x)| d x<\infty$.

In $[5,6]$, the threshold behavior has been studied as a general perturbation phenomenon and some general results on existence and asymptotic behavior of eigenvalues for selfadjoint operators $A+\lambda B$ have been obtained. The main tool was the so-called BirmanSchwinger principle. Klaus [6] has also applied these results to several special cases. One of them has been concerned with the Hamiltonian $-\frac{d^{2}}{d x^{2}}+U+\lambda V$. If a certain relation

2020 Mathematics Subject Classification. Primary 34L40, 34B09; Secondary 81Q10.

Key words and phrases. 1D Schrödinger operator, coupling constant threshold, negative eigenvalue, zero-energy resonance, half-bound state. 
between the potentials $U$ and $V$ holds, then the operator has a small negative-energy bound state (not necessarily a unique one) in the limit of weak coupling. Namely, it has been proved that the operator has the coupling constant threshold $\lambda=0$, if the unperturbed operator $-\frac{d^{2}}{d x^{2}}+U$ possesses a zero-energy resonance with a half-bound state $u$ and $\int_{\mathbb{R}} V u^{2} d x<0$. Among the negative eigenvalues there exists only one that is absorbed by the continuous spectrum as $\lambda \rightarrow 0$. A unique threshold eigenvalue $e_{\lambda}$ is analytic at $\lambda=0$ and obeys

$$
\sqrt{-e_{\lambda}}=-\frac{\lambda}{u_{-}^{2}+u_{+}^{2}} \int_{\mathbb{R}} V u^{2} d x+O\left(\lambda^{2}\right)
$$

as $\lambda \rightarrow 0$, where $u_{ \pm}=\lim _{x \rightarrow \pm \infty} u(x)$. If $\int_{\mathbb{R}} V u^{2} d x=0$ and the support of $V$ lies between two consecutive zeros of $u$, then there exists a bound state near zero for all small enough $\lambda$ (positive and negative). Finally, if $\int_{\mathbb{R}} V u^{2} d x>0$, then the operator has no bound state and therefore $\lambda=0$ is not a coupling constant threshold. We will give the precise definitions of the zero-energy resonances, half-bound states, and coupling constant threshold in the next section.

One of the motivations for writing this article was the desire to improve approximation (2). As another motivation for investigating the threshold behavior of eigenvalues, we mention applications of this phenomenon to the study of the stability of solutions for the Korteweg-de Vries equation [21] and the existence of 'breathers' (the localized periodic solutions) for discrete nonlinear Schrödinger systems [22, 23].

In this paper, we consider a more general class of Schrodinger operators

$$
H_{\lambda}=-\frac{d^{2}}{d x^{2}}+U+\lambda V_{\lambda}, \quad \operatorname{dom} H_{\lambda}=W_{2}^{2}(\mathbb{R})
$$

with nonlinear dependence on the positive parameter $\lambda$. We analyze the existence of negative eigenvalues and their threshold behavior. Here $U$ and $V_{\lambda}$ are functions of compact support and $V_{\lambda}=V+\lambda V_{1}+o(\lambda)$ as $\lambda \rightarrow 0$. The spectrum of $H_{\lambda}$ consists of the essential spectrum $[0, \infty)$ and possibly a finite number of negative eigenvalues. Under certain conditions on the potentials $U, V$ and $V_{1}$ the operator $H_{\lambda}$ has a negative eigenvalue $e_{\lambda}$ that is absorbed at the bottom of the essential spectrum as $\lambda$ goes to zero. The threshold eigenvalue may or may not be the ground state. We examine the asymptotic behavior of $e_{\lambda}$ as $\lambda \rightarrow 0$ and compute the two term asymptotic formula which in particular improves the approximation (2). For the case $U=0$ and $V_{\lambda}=V$, our asymptotics turns into the Abarbanel-Callan-Goldberger formula.

The threshold behavior of eigenvalues for operators $-\frac{d^{2}}{d x^{2}}+U+\lambda \alpha_{\lambda} V\left(\alpha_{\lambda} \cdot\right)$, where the positive sequence $\alpha_{\lambda}$ converges to a finite or infinite limit as $\lambda \rightarrow 0$, has recently been studied in [7]. These results gives us an example of the non-analytic threshold behavior of negative eigenvalues.

The question of how negative eigenvalues are absorbed in the bottom of the essential spectrum has been discussed by many authors [8-18]. The Hamiltonians with periodic potentials perturbed by short range ones and the threshold phenomena in gaps of the continuous spectrum were studied in $[6,19,20]$.

\section{Main Results}

We start with some definitions. Let $A$ and $B_{\lambda}$ be self-adjoint operators and $B_{\lambda}$ be relatively $A$-compact for all $\lambda>0$; then $\sigma_{\text {ess }}\left(A+B_{\lambda}\right)=\sigma_{\text {ess }}(A)$. Suppose that the interval $(a, b)$ is a gap in the spectrum of $A$. If we can find an eigenvalue $e_{\lambda}$ of $A+B_{\lambda}$ in $(a, b)$ for all $\lambda>0$ with the property that $e_{\lambda} \rightarrow a$ or $e_{\lambda} \rightarrow b$ as $\lambda \rightarrow 0$, then we call $\lambda=0$ the coupling constant threshold. So the eigenvalue $e_{\lambda}$ is absorbed by the continuous spectrum at "time" $\lambda=0$. 
We say operator $-\frac{d^{2}}{d x^{2}}+U$ possesses a zero-energy resonance if there exists a non trivial solution $u$ of the equation

$$
-u^{\prime \prime}+U u=0
$$

that is bounded on the whole line. We then call $u$ the half-bound state. Any half-bound state $u$ possesses finite limits $\lim _{x \rightarrow \pm \infty} u(x)$, because $u$ is constant outside the support of $U$; both the limits are different from zero. Since a half-bound state is defined up to a scalar multiplier, we say a half-bound state $u$ is normalized if $\lim _{x \rightarrow-\infty} u(x)=1$. Let $\theta$ hereafter denote the limit of the normalized half-bound state as $x \rightarrow+\infty$, i.e., $\theta:=\lim _{x \rightarrow+\infty} u(x)$. We also introduce the function

$$
\Theta(x)= \begin{cases}1 & \text { if } x<0 \\ \theta & \text { if } x>0 .\end{cases}
$$

Assume $u_{1}$ is a solution of (4) such that $u_{1}(x)=x$ to the left of the support of $U$. Then $u$ and $u_{1}$ are linearly independent solutions of (4) and we will show below that there exists a constant $\theta_{1}$ such that $u_{1}(x)=\theta^{-1} x+\theta_{1}$ for all $x$ large enough (see Fig. 1 ). Let $v_{*}$ be a solution of $-v^{\prime \prime}+U v=-V u$ which vanishes to the left of the supports of $U$ and $V$.
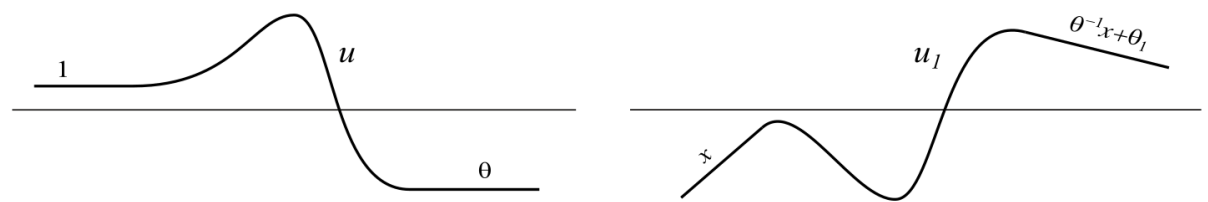

Figure 1. Plots of normalized half-bound state $u$ and solution $u_{1}$.

Here and subsequently, $\|\cdot\|$ stands for the norm in $L_{2}(\mathbb{R})$.

Theorem 1. Suppose that $U, V$ and $V_{1}$ are functions of compact support belonging to $L^{\infty}(\mathbb{R})$, and $\left\|V_{\lambda}-V-\lambda V_{1}\right\|=o(\lambda)$ as $\lambda \rightarrow 0$. Assume operator $-\frac{d^{2}}{d x^{2}}+U$ has a zero-energy resonance with normalized half-bound state $u$. If

$$
\int_{\mathbb{R}} V u^{2} d x<0
$$

then operator $H_{\lambda}=-\frac{d^{2}}{d x^{2}}+U+\lambda V_{\lambda}$ possesses the coupling constant threshold $\lambda=0$, i.e., for all small positive $\lambda$ there exists a negative eigenvalue $e_{\lambda}$ of $H_{\lambda}$ such that $e_{\lambda} \rightarrow 0$ as $\lambda \rightarrow 0$. Moreover the threshold eigenvalue $e_{\lambda}$ has the asymptotic expansion $e_{\lambda}=$ $-\lambda^{2}\left(\omega_{0}+\omega_{1} \lambda+o(\lambda)\right)^{2}$ as $\lambda \rightarrow 0$, where

$$
\begin{gathered}
\omega_{0}=\frac{1}{\theta^{2}+1} \int_{\mathbb{R}} V u^{2} d x \\
\omega_{1}=\frac{1}{\theta^{2}+1}\left(\int_{\mathbb{R}} V\left(v_{*}+\omega_{0}\left(\theta^{2}-1\right) u_{1}\right) u d x\right. \\
\left.+\omega_{0}^{2} \int_{\mathbb{R}}\left(u^{2}-\Theta^{2}\right) d x-\omega_{0}^{2} \theta^{3} \theta_{1}+\int_{\mathbb{R}} V_{1} u^{2} d x\right) .
\end{gathered}
$$

The threshold phenomenon is also possible if inequality (5) turns into the equality. In this case the absorption of the eigenvalue at the bottom of $\sigma_{\text {ess }}\left(H_{\lambda}\right)$ occurs with the rate $O\left(\lambda^{4}\right)$ as $\lambda \rightarrow 0$. 
Theorem 2. Under the assumptions of Theorem 1, we suppose that

$$
\int_{\mathbb{R}} V u^{2} d x=0 .
$$

Then the operator $H_{\lambda}$ has the coupling constant threshold $\lambda=0$, if

$$
\int_{\mathbb{R}}\left(V v_{*}+V_{1} u\right) u d x<0 .
$$

Moreover the threshold eigenvalue $e_{\lambda}$ admits the asymptotics

$$
e_{\lambda}=-\frac{\lambda^{4}}{\left(\theta^{2}+1\right)^{2}}\left(\int_{\mathbb{R}} V v_{*} u d x+\int_{\mathbb{R}} V_{1} u^{2} d x\right)^{2}+o\left(\lambda^{4}\right) \quad \text { as } \quad \lambda \rightarrow 0 .
$$

Return now to operator family $-\frac{d^{2}}{d x^{2}}+U+\lambda V$ studied in [6].

Corollary 1. Assume the operator $-\frac{d^{2}}{d x^{2}}+U$ has a zero-energy resonance with half-bound state $u$. If

$$
\int_{\mathbb{R}} V u^{2} d x<0
$$

then $-\frac{d^{2}}{d x^{2}}+U+\lambda V$ possesses the coupling constant threshold $\lambda=0$ and a negative eigenvalue $e_{\lambda}$ admits the asymptotics

$$
e_{\lambda}=-\lambda^{2}\left(\omega_{0}+\lambda \omega_{1}+o(\lambda)\right)^{2}
$$

where $\omega_{0}$ is given by (6) and

$$
\omega_{1}=\frac{1}{\theta^{2}+1}\left(\int_{\mathbb{R}} V\left(v_{*}+\omega_{0}\left(\theta^{2}-1\right) u_{1}\right) u d x+\omega_{0}^{2} \int_{\mathbb{R}}\left(u^{2}-\Theta^{2}\right) d x-\omega_{0}^{2} \theta^{3} \theta_{1}\right) .
$$

If $V$ is different from zero and

$$
\int_{\mathbb{R}} V u^{2} d x=0
$$

then the operator $-\frac{d^{2}}{d x^{2}}+U+\lambda V$ has a negative eigenvalue $e_{\lambda}$ with the asymptotics

$$
e_{\lambda}=-\frac{\lambda^{4}}{\left(u_{-}^{2}+u_{+}^{2}\right)^{2}}\left(\iint_{\mathbb{R}^{2}} V(x) u(x) \mathcal{E}_{U}(x-y) V(y) u(y) d x d y+o(1)\right)^{2},
$$

where $\mathcal{E}_{U}$ is the fundamental solution for $\frac{d^{2}}{d x^{2}}-U$ which vanishes to the left of $\operatorname{supp} U$.

Proof. Most of the proof follows from the previous theorems, assuming $V_{\lambda}=V$ for all $\lambda$. We are left with the task of deriving (12). If (11) holds, then $\omega_{0}=0$ and

$$
\omega_{1}=\frac{1}{\theta^{2}+1} \int_{\mathbb{R}} V v_{*} u d x .
$$

Recall that $v_{*}$ solves equation $v_{*}^{\prime \prime}-U v_{*}=V u$ and vanishes to the left of the supports of $U$ and $V$. Then $v_{*}$ can be represented as the convolution $\mathcal{E}_{U} *(V u)$. Hence

$$
\begin{aligned}
\int_{\mathbb{R}} V v_{*} u d x & =\int_{\mathbb{R}} V(x) u(x)\left(\mathcal{E}_{U} * V u\right)(x) d x \\
& =\iint_{\mathbb{R}^{2}} V(x) u(x) \mathcal{E}_{U}(x-y) V(y) u(y) d x d y .
\end{aligned}
$$

Substituting (14) into (13) finishes up the proof. 
Remark 1. Klaus did not use the notion of a normalized half-bound state. To agree the asymptotic formulas, we rewrite $\omega_{0}$ and $\omega_{1}$ in (10) in terms of an arbitrary half-bound state $u$ for which $\lim _{x \rightarrow \pm \infty} u(x)=u_{ \pm}$. Then in notation of [6] we obtain

$$
\begin{gathered}
\omega_{0}=\frac{1}{u_{-}^{2}+u_{+}^{2}} \int_{\mathbb{R}} V u^{2} d x \\
\omega_{1}=\frac{1}{u_{-}^{2}+u_{+}^{2}}\left(u_{-} \int_{\mathbb{R}} V\left(v_{*}+\frac{\omega_{0}\left(u_{+}^{2}-u_{-}^{2}\right)}{u_{-}^{2}} u_{1}\right) u d x\right. \\
\left.+\omega_{0}^{2} \int_{\mathbb{R}}\left(u^{2}-\Xi^{2}\right) d x-\omega_{0}^{2} \theta_{1} \frac{u_{+}^{3}}{u_{-}}\right),
\end{gathered}
$$

where $\Xi(x)=u_{-}$for $x<0$ and $\Xi(x)=u_{+}$for $x>0$.

Let us compare our results with those of Simon when the unperturbed operator is the free Schrödinger operator.

Corollary 2. Assume that $U=0$. If the mean value of $V$ is negative, i.e.,

$$
\int_{\mathbb{R}} V d x<0
$$

then $H_{\lambda}=-\frac{d^{2}}{d x^{2}}+\lambda V_{\lambda}$ has a negative eigenvalue of the form

$$
e_{\lambda}=-\lambda^{2}\left(\omega_{0}+\lambda \omega_{1}+o(\lambda)\right)^{2}
$$

as $\lambda$ tends to zero, where

$$
\omega_{0}=\frac{1}{2} \int_{\mathbb{R}} V d x, \quad \omega_{1}=\frac{1}{4} \iint_{\mathbb{R}^{2}} V(x)|x-y| V(y) d x d y+\frac{1}{2} \int_{\mathbb{R}} V_{1} d x .
$$

In the case $V_{\lambda}=V$, this asymptotic formula coincides with (1).

Proof. The trivial potential $U=0$ has a zero-energy resonance with half-bound state $u=1$; then $\theta=1$ and $\Theta(x)=1$ for all $x \in \mathbb{R}$. In addition, we have $\theta_{1}=0$, because equation $u^{\prime \prime}=0$ possesses the solution $u_{1}=x$. Therefore condition (5) becomes (15), and (6), (7) simplify to read

$$
\omega_{0}=\frac{1}{2} \int_{\mathbb{R}} V d x, \quad \omega_{1}=\frac{1}{2} \int_{\mathbb{R}} V v_{*} d x+\frac{1}{2} \int_{\mathbb{R}} V_{1} d x .
$$

The fundamental solution $\mathcal{E}_{0}(x)=\frac{1}{2}(|x|+x)$ for the differential operator $\frac{d^{2}}{d x^{2}}$ vanishes for $x<0$. As in Corollary 1 , we derive

$$
\begin{aligned}
& \int_{\mathbb{R}} V(x) v_{*}(x) d x=\int_{\mathbb{R}} V(x)\left(\mathcal{E}_{0} * V\right)(x) d x \\
&=\frac{1}{2} \iint_{\mathbb{R}^{2}} V(x)|x-y| V(y) d x d y+\frac{1}{2} \iint_{\mathbb{R}^{2}} V(x)(x-y) V(y) d x d y \\
& \quad=\frac{1}{2} \iint_{\mathbb{R}^{2}} V(x)|x-y| V(y) d x d y,
\end{aligned}
$$

because $\iint_{\mathbb{R}^{2}} f(x)(x-y) f(y) d x d y=0$ for any $f$, for which the integral exists. This gives the second equality in (16), and the proof is complete.

Corollary 3. Assume that $U=0$ and $V$ is different from zero. If

$$
\int_{\mathbb{R}} V d x=0
$$


then for all nonzero $\lambda$, positive or negative, the operator $H_{\lambda}=-\frac{d^{2}}{d x^{2}}+\lambda V_{\lambda}$ possesses an eigenvalue $e_{\lambda}$ having the asymptotics

$$
e_{\lambda}=-\frac{\lambda^{4}}{16}\left(\iint_{\mathbb{R}^{2}} V(x)|x-y| V(y) d x d y+o(1)\right)^{2}
$$

as $\lambda \rightarrow 0$. This asymptotic formula can be also written in the form

$$
e_{\lambda}=-\frac{\lambda^{4}}{4}\left(\int_{\mathbb{R}}\left(\int_{-\infty}^{x} V(y) d y\right)^{2} d x+o(1)\right)^{2} .
$$

This assertion will be proved in Section 4 .

\section{Preliminaries}

We first record some technical facts. Assume, without loss of generality, the supports of potentials $U$ and $V_{\lambda}$ lie within $\mathcal{I}=(-\ell, \ell)$ for $\lambda$ small enough. Then a half-bound state of operator $-\frac{d^{2}}{d x^{2}}+U$ is constant outside $\mathcal{I}$ and its restriction to $\mathcal{I}$ is a non-trivial solution of the problem

$$
-u^{\prime \prime}+U u=0, \quad t \in \mathcal{I}, \quad u^{\prime}(-\ell)=0, \quad u^{\prime}(\ell)=0 .
$$

Moreover, if $u$ is the normalized half-bound state, then $u(-\ell)=1$ and $u(\ell)=\theta$.

Proposition 1. Assume that $h$ belongs to $L_{2}(\mathcal{I})$ and $\gamma$ is a real number. Let $w$ be a solution of the Cauchy problem

$$
-w^{\prime \prime}+U w=h, \quad t \in \mathcal{I}, \quad w(-\ell)=0, \quad w^{\prime}(-\ell)=\gamma .
$$

If $-\frac{d^{2}}{d x^{2}}+U$ has a zero-energy resonance with normalized half-bound state $u$, then

$$
\theta w^{\prime}(\ell)=\gamma-\int_{-\ell}^{\ell} h u d x .
$$

In addition, this solution obeys the estimate

$$
\|w\|_{C^{1}(\mathcal{I})} \leq C\left(|\gamma|+\|h\|_{L_{2}(\mathcal{I})}\right)
$$

for some positive $C$ being independent of $\gamma$ and $h$.

Proof. Since $u(-\ell)=1$ and $u(\ell)=\theta,(20)$ can be easily obtained by multiplying the equation in (19) by $u$ and integrating by parts. Next, application of the variation of parameters method yields

$$
w(x)=\gamma\left(u_{1}(x)+\ell u(x)\right)+\int_{-\ell}^{x} k(x, s) h(s) d s,
$$

where $k(x, s)=u(x) u_{1}(s)-u(s) u_{1}(x)$. Under the assumptions made on potential $U, u$ and $u_{1}$ belong to $W_{2}^{2}(\mathcal{I})$; consequently $u, u_{1} \in C^{1}(\mathcal{I})$ by the Sobolev embedding theorem. From this and the representation of the first derivative

$$
w^{\prime}(x)=\gamma\left(u_{1}^{\prime}(x)+\ell u^{\prime}(x)\right)+\int_{-\ell}^{x} \frac{\partial k}{\partial x}(x, s) h(s) d s
$$

we have $|w(x)|+\left|w^{\prime}(x)\right| \leq|\gamma|\left(\left\|u_{1}\right\|_{C^{1}(\mathcal{I})}+|\ell|\|u\|_{C^{1}(\mathcal{I})}\right)+c_{1}\|k\|_{C^{1}(\mathcal{I} \times \mathcal{I})}\|h\|_{L_{2}(\mathcal{I})} \leq C(|\gamma|+$ $\left.\|h\|_{L_{2}(\mathcal{I})}\right)$ for $x \in \mathcal{I}$, which completes the proof.

Proposition 2. Let $u_{1}$ be the solution of (4) as described in Section 2. Then for some constant $\theta_{1}$ we have $u_{1}(x)=\theta^{-1} x+\theta_{1}$ for all $x>\ell$. 
Proof. The function $v=u_{1}+\ell u$ solves the Cauchy problem

$$
-v^{\prime \prime}+U v=0, \quad t \in \mathcal{I}, \quad v(-\ell)=0, \quad v^{\prime}(-\ell)=1
$$

and therefore $u_{1}^{\prime}(\ell)=\theta^{-1}$ by $(20)$. Hence $u_{1}(x)=\theta^{-1} x+\theta_{1}$ for some $\theta_{1}$ and all $x>\ell$, which is the desired conclusion.

Our method is different from that of Simon and Klaus. We don't use the BirmanSchwinger principle. To prove the main results, we use the asymptotic method of quasimodes or in other words of "almost" eigenvalues and eigenfunctions. Let $A$ be a selfadjoint operator in a Hilbert space $L$. We say a pair $(\mu, \phi) \in \mathbb{R} \times \operatorname{dom} A$ is a quasimode of $A$ with accuracy $\delta$, if $\|\phi\|_{L}=1$ and $\|(A-\mu I) \phi\|_{L} \leq \delta$.

Lemma 1 ([24, p. 139]). Assume $(\mu, \phi)$ is a quasimode of $A$ with accuracy $\delta>0$ and the spectrum of $A$ is discrete in the interval $[\mu-\delta, \mu+\delta]$. Then there exists an eigenvalue $\lambda$ of $A$ such that $|\lambda-\mu| \leq \delta$.

Proof. If $\mu \in \sigma(A)$, then $\lambda=\mu$. Otherwise the distance $d_{\mu}$ from $\mu$ to the spectrum of $A$ can be computed as

$$
d_{\mu}=\left\|(A-\mu I)^{-1}\right\|^{-1}=\inf _{\psi \neq 0} \frac{\|\psi\|_{L}}{\left\|(A-\mu I)^{-1} \psi\right\|_{L}},
$$

where $\psi$ is an arbitrary vector of $L$. Taking $\psi=(A-\mu I) \phi$, we deduce

$$
d_{\mu} \leq \frac{\|(A-\mu I) \phi\|_{L}}{\|\phi\|_{L}} \leq \delta,
$$

from which the assertion follows.

\section{Proof of Main Results}

4.1. Proof of Theorem 1. In order to prove the existence of a negative eigenvalue for $H_{\lambda}$, we will construct a quasimode $\left(-\omega_{\lambda}^{2}, \phi_{\lambda}\right)$ of $H_{\lambda}$ as follows. Suppose that $-\frac{d^{2}}{d x^{2}}+U$ has a zero-energy resonance with normalized half-bound state $u$. We assume $\omega_{\lambda}=$ $\lambda\left(\omega_{0}+\lambda \omega_{1, \lambda}+\lambda^{2} \omega_{2, \lambda}\right)$ and $\phi_{\lambda}=\psi_{\lambda} /\left\|\psi_{\lambda}\right\|$, where

$$
\psi_{\lambda}(x)= \begin{cases}e^{-\omega_{\lambda}(x+\ell)} & \text { for } x<-\ell, \\ u(x)+\lambda v_{1}(x)+\lambda^{2} v_{2, \lambda}(x)+\lambda^{3} v_{3, \lambda}(x) & \text { for }|x|<\ell, \\ a_{\lambda} e^{\omega_{\lambda}(x-\ell)}+b_{\lambda} \rho(x-\ell) & \text { for } x>\ell .\end{cases}
$$

The functions $v_{1}, v_{2, \lambda}$ and $v_{3, \lambda}$ are solutions of the problems

$$
\begin{aligned}
& -v_{1}^{\prime \prime}+U v_{1}=-V u, \quad v_{1}(-\ell)=0, \quad v_{1}^{\prime}(-\ell)=-\omega_{0} ; \\
& \left\{\begin{array}{r}
-v_{2}^{\prime \prime}+U v_{2}=-V v_{1}-\left(V_{1}+g_{\lambda}\right) u \\
v_{2}(-\ell)=0, \quad v_{2}^{\prime}(-\ell)=-\omega_{1, \lambda} ;
\end{array}\right. \\
& -v_{3}^{\prime \prime}+U v_{3}=-f_{3, \lambda}, \quad v_{3}(-\ell)=0, \quad v_{3}^{\prime}(-\ell)=-\omega_{2, \lambda}
\end{aligned}
$$

respectively. Here we set $g_{\lambda}=\lambda^{-1}\left(V_{\lambda}-V-\lambda V_{1}\right)$ and

$$
f_{3, \lambda}=V v_{2, \lambda}+\left(V_{1}+\omega_{0}^{2}+g_{\lambda}\right) v_{1}+2 \omega_{0} \omega_{1, \lambda} u .
$$

We also presume that $\omega_{1, \lambda}$ and $\omega_{2, \lambda}$ have finite limits as $\lambda \rightarrow 0$. The function $\rho$ is smooth in $\mathbb{R} \backslash\{0\}, \rho(x)=0$ for $x \leq 0$ and $x \geq 1$, and $\rho^{\prime}(+0)=1$. In addition, $\rho^{\prime \prime}$ is bounded in $[0,1]$. Hence $\rho$ is continuous at $x=0$, but the first derivative $\rho^{\prime}$ has the unit jump at this point. This function corrects the discontinuity of $\psi_{\lambda}^{\prime}$ at $x=\ell$.

Let us first show that constants $\omega_{0}, \omega_{1, \lambda}, \omega_{2, \lambda}, a_{\lambda}$ and $b_{\lambda}$ in $(22)$ can be chosen so that $\psi_{\lambda}$ will belong to dom $H_{\lambda}$. First of all, the $L_{2}(\mathbb{R})$-norm of $\psi_{\lambda}$ is finite if and only if $\omega_{\lambda}<0$; therefore we must impose the conditions $\omega_{0}<0$ (the case $\omega_{0}=0$ will be treated in Theorem 2). Note that $u$ and $v_{k}$ belong to the Sobolev space $W_{2}^{2}(\mathcal{I})$ as solutions of 
the equation $-y^{\prime \prime}+U y=f$ with $f \in L_{2}(\mathcal{I})$. By construction, $\psi_{\lambda}$ and its first derivative are continuous at $x=-\ell$, then it is enough to ensure the continuous differentiability of $\psi_{\lambda}$ at $x=\ell$.

Since $\psi_{\lambda}(\ell+0)-\psi_{\lambda}(\ell-0)=\theta+\lambda v_{1}(\ell)+\lambda^{2} v_{2, \lambda}(\ell)+\lambda^{3} v_{3, \lambda}(\ell)-a_{\lambda}$, we set

$$
a_{\lambda}=\theta+\lambda v_{1}(\ell)+\lambda^{2} v_{2, \lambda}(\ell)+\lambda^{3} v_{3, \lambda}(\ell) .
$$

To see this, we calculate

$$
\begin{aligned}
\psi_{\lambda}^{\prime}(\ell+0)- & \psi_{\lambda}^{\prime}(\ell-0)=\omega_{\lambda} a_{\lambda}+b_{\lambda} \rho^{\prime}(0)-\lambda v_{1}^{\prime}(\ell)-\lambda^{2} v_{2, \lambda}^{\prime}(\ell)-\lambda^{3} v_{3, \lambda}^{\prime}(\ell) \\
= & \lambda\left(\omega_{0}+\lambda \omega_{1, \lambda}+\lambda^{2} \omega_{2, \lambda}\right)\left(\theta+\lambda v_{1}(\ell)+\lambda^{2} v_{2, \lambda}(\ell)+\lambda^{3} v_{3, \lambda}(\ell)\right) \\
& +b_{\lambda}-\lambda v_{1}^{\prime}(\ell)-\lambda^{2} v_{2, \lambda}^{\prime}(\ell)-\lambda^{3} v_{3, \lambda}^{\prime}(\ell) \\
= & \lambda\left(\omega_{0} \theta-v_{1}^{\prime}(\ell)\right)+\lambda^{2}\left(\omega_{1, \lambda} \theta+\omega_{0} v_{1}(\ell)-v_{2, \lambda}^{\prime}(\ell)\right) \\
& +\lambda^{3}\left(\omega_{2, \lambda} \theta+\omega_{1, \lambda} v_{1}(\ell)+\omega_{0} v_{2, \lambda}(\ell)-v_{3, \lambda}^{\prime}(\ell)\right)+b_{\lambda} \\
& +\lambda^{4}\left(\omega_{0} v_{3, \lambda}(\ell)+\omega_{1, \lambda}\left(v_{2, \lambda}(\ell)+\lambda v_{3, \lambda}(\ell)\right)\right. \\
& \left.+\omega_{2, \lambda}\left(v_{1}(\ell)+\lambda v_{2, \lambda}(\ell)+\lambda^{2} v_{3, \lambda}(\ell)\right)\right) .
\end{aligned}
$$

In order to achieve $\psi_{\lambda}^{\prime}(\ell+0)=\psi_{\lambda}^{\prime}(\ell-0)$, we assume

$$
\begin{gathered}
\omega_{0}=\theta^{-1} v_{1}^{\prime}(\ell), \quad \omega_{1, \lambda}=\theta^{-1}\left(v_{2, \lambda}^{\prime}(\ell)-\omega_{0} v_{1}(\ell)\right), \\
\omega_{2, \lambda}=\theta^{-1}\left(v_{3, \lambda}^{\prime}(\ell)-\omega_{0} v_{2, \lambda}(\ell)-\omega_{1, \lambda} v_{1}(\ell)\right), \\
b_{\lambda}=-\left.\lambda^{4}\left(\omega_{0} v_{3, \lambda}+\omega_{1, \lambda}\left(v_{2, \lambda}+\lambda v_{3, \lambda}\right)+\omega_{2, \lambda}\left(v_{1}+\lambda v_{2, \lambda}+\lambda^{2} v_{3, \lambda}\right)\right)\right|_{x=\ell .}
\end{gathered}
$$

On the other hand, applying Proposition 1 to problems (23)-(25), we deduce

$$
\begin{gathered}
\theta v_{1}^{\prime}(\ell)=-\omega_{0}+\int_{-\ell}^{\ell} V u^{2} d x, \quad \theta v_{3, \lambda}^{\prime}(\ell)=-\omega_{2, \lambda}+\int_{-\ell}^{\ell} f_{3, \lambda} u d x, \\
\theta v_{2, \lambda}^{\prime}(\ell)=-\omega_{1, \lambda}+\int_{-\ell}^{\ell} V v_{1} u d x+\int_{-\ell}^{\ell}\left(V_{1}+\omega_{0}^{2}+g_{\lambda}\right) u^{2} d x .
\end{gathered}
$$

Then combining (27), (28), (30) and (31) yields

$$
\begin{gathered}
\omega_{0}=\frac{1}{\theta^{2}+1} \int_{-\ell}^{\ell} V u^{2} d x \\
\omega_{1, \lambda}=\frac{1}{\theta^{2}+1}\left(\int_{-\ell}^{\ell} V v_{1} u d x-\theta \omega_{0} v_{1}(\ell)+\int_{-\ell}^{\ell}\left(V_{1}+\omega_{0}^{2}+g_{\lambda}\right) u^{2} d x\right), \\
\omega_{2, \lambda}=\frac{1}{\theta^{2}+1}\left(\int_{-\ell}^{\ell} f_{3, \lambda} u d x-\theta\left(\omega_{0} v_{2, \lambda}(\ell)+\omega_{1, \lambda} v_{1}(\ell)\right)\right) .
\end{gathered}
$$

Since $V$ has a compact support, $\omega_{0}$ does not depend on $\ell$ and can be finally written in the form

$$
\omega_{0}=\frac{1}{\theta^{2}+1} \int_{\mathbb{R}} V u^{2} d x
$$

Moreover $\omega_{0}$ is negative if condition (5) holds; then $\omega_{\lambda}$ is negative for all $\lambda$ small enough and therefore $\psi_{\lambda} \in L_{2}(\mathbb{R})$.

The function $g_{\lambda}$ has an infinitely small $L_{2}(\mathbb{R})$-norm as $\lambda \rightarrow 0$, since

$$
\left\|V_{\lambda}-V-\lambda V_{1}\right\|=o(\lambda) \quad \text { as } \quad \lambda \rightarrow 0 .
$$


Consequently there exists limit $\omega_{1}=\lim _{\lambda \rightarrow 0} \omega_{1, \lambda}$, where

$$
\omega_{1}=\frac{1}{\theta^{2}+1}\left(\int_{-\ell}^{\ell} V v_{1} u d x-\theta \omega_{0} v_{1}(\ell)+\int_{-\ell}^{\ell}\left(V_{1}+\omega_{0}^{2}\right) u^{2} d x\right) .
$$

But it is not obvious that $\omega_{1}$ does not depend on $\ell$, because the right hand side of (34) contains the integrand $\omega_{0}^{2} u^{2}$ without a compact support as well as the solution $v_{1}$ of $(23)$ which depends on $\ell$. We first note that $u^{2}-\Theta^{2}$ is a function of compact support. Then we have

$$
\int_{-\ell}^{\ell} u^{2} d x=\int_{-\ell}^{\ell}\left(u^{2}-\Theta^{2}\right) d x+\int_{-\ell}^{\ell} \Theta^{2} d x=\int_{\mathbb{R}}\left(u^{2}-\Theta^{2}\right) d x+\ell\left(\theta^{2}+1\right) .
$$

Next, $v_{1}$ can be written as $v_{1}=v_{*}-\omega_{0}\left(u_{1}+\ell u\right)$, where $v_{*}$ is the solution of the Cauchy problem $-v_{*}^{\prime \prime}+U v_{*}=-V u, v_{*}(-\ell)=0, v_{*}^{\prime}(-\ell)=0$. Invoking (33), we derive

$$
\begin{aligned}
\int_{-\ell}^{\ell} V v_{1} u d x & =\int_{-\ell}^{\ell} V\left(v_{*}-\omega_{0} u_{1}\right) u d x-\omega_{0} \ell \int_{-\ell}^{\ell} V u^{2} d x \\
& =\int_{\mathbb{R}} V\left(v_{*}-\omega_{0} u_{1}\right) u d x-\omega_{0}^{2} \ell\left(\theta^{2}+1\right) .
\end{aligned}
$$

In order to compute $v_{1}(\ell)$, we multiply the equation in (23) by $u_{1}$ and integrate by parts twice $\left.\left(v_{1}^{\prime} u_{1}-v_{1} u_{1}^{\prime}\right)\right|_{-\ell} ^{\ell}=\int_{-\ell}^{\ell} V u u_{1} d x$. Since $u_{1}(-\ell)=-\ell, u_{1}(\ell)=\ell \theta^{-1}+\theta_{1}, u_{1}^{\prime}(\ell)=\theta^{-1}$ and $v_{1}^{\prime}(\ell)=\omega_{0} \theta$, we obtain

$$
v_{1}(\ell)=\omega_{0} \theta^{2} \theta_{1}-\theta \int_{\mathbb{R}} V u_{1} u d x .
$$

Substitute (35)-(37) into (34), to find

$$
\omega_{1}=\frac{1}{\theta^{2}+1}\left(\int_{\mathbb{R}} V v_{0} u d x+\int_{\mathbb{R}} V_{1} u^{2} d x+\omega_{0}^{2} \int_{\mathbb{R}}\left(u^{2}-\Theta^{2}\right) d x-\omega_{0}^{2} \theta^{3} \theta_{1}\right),
$$

where $v_{0}=v_{*}+\omega_{0}\left(\theta^{2}-1\right) u_{1}$. Hence $\omega_{1}$ does not depend on $\ell$ either. A similar arguments can be applied to $\omega_{2, \lambda}$. Therefore $\psi_{\lambda}$ belongs to dom $H_{\lambda}$ by our choice of $\omega_{0}, \omega_{1, \lambda}, \omega_{2, \lambda}$, $a_{\lambda}$ and $b_{\lambda}$.

Proposition 3. There exist constants $c$ and $C$ such that

$$
c \omega_{\lambda}^{-1 / 2} \leq\left\|\psi_{\lambda}\right\| \leq C \omega_{\lambda}^{-1 / 2}
$$

Proof. We first note that the solutions $v_{2, \lambda}$ and $v_{3, \lambda}$ are bounded in $L_{2}(\mathbb{R})$ uniformly on $\lambda$. In addition, by Proposition 1 we have

$$
\begin{gathered}
\left\|v_{2, \lambda}\right\|_{C^{1}(\mathcal{I})} \leq C\left(\left|\omega_{1, \lambda}\right|+\left\|V v_{1}+\left(V_{1}+g_{\lambda}\right) u\right\|_{L_{2}(\mathcal{I})}\right) \leq c_{1}, \\
\left\|v_{3, \lambda}\right\|_{C^{1}(\mathcal{I})} \leq C\left(\left|\omega_{2, \lambda}\right|+\left\|f_{3, \lambda}\right\|_{L_{2}(\mathcal{I})}\right) \leq c_{2}
\end{gathered}
$$

where $c_{1}$ and $c_{2}$ are independent of $\lambda$. Combining these bounds with (26) and (29) yields

$$
\left|a_{\lambda}\right| \leq c_{3}, \quad\left|b_{\lambda}\right| \leq c_{3} \lambda^{4}
$$

Therefore the main contribution as $\lambda \rightarrow 0$ to the norm of $\psi_{\lambda}$ is given by the exponents $e^{ \pm \omega_{\lambda}(x \mp \ell)}$. A direct calculation verifies $\left\|e^{-\omega_{\lambda}(x+\ell)}\right\|_{L_{2}(-\infty,-\ell)}=\left(2 \omega_{\lambda}\right)^{-1 / 2}$ and $\left\|e^{\omega_{\lambda}(x-\ell)}\right\|_{L_{2}(\ell,+\infty)}=\left(2 \omega_{\lambda}\right)^{-1 / 2}$. Hence $\left\|\psi_{\lambda}\right\| \sim a \omega_{\lambda}^{-1 / 2}$ as $\lambda \rightarrow 0$. In particular, $\left\|\psi_{\lambda}\right\| \sim a_{0} \lambda^{-1 / 2}$ if $\omega_{0} \neq 0$ and $\left\|\psi_{\lambda}\right\| \sim a_{1} \lambda^{-1}$ if $\omega_{0}=0$.

Lemma 2. The pair $\left(-\omega_{\lambda}^{2}, \phi_{\lambda}\right)$ is a quasimode of $H_{\lambda}$ with the accuracy $o\left(\lambda^{9 / 2}\right)$ as $\lambda \rightarrow 0$. 
Proof. Let $r_{\lambda}=\left(H_{\lambda}+\omega_{\lambda}^{2} I\right) \psi_{\lambda}$. Then $\left(H_{\lambda}+\omega_{\lambda}^{2} I\right) \phi_{\lambda}=\left\|\psi_{\lambda}\right\|^{-1} r_{\lambda}$. We must estimate the $L_{2}$-norm of $r_{\lambda}$. Since $e^{ \pm \omega_{\lambda}(x \mp \ell)}$ are exact solutions of $-\psi^{\prime \prime}+\omega_{\lambda}^{2} \psi=0$ and $\operatorname{supp} \rho=[0,1]$, we have

$$
r_{\lambda}(x)=-b_{\lambda}\left(\rho^{\prime \prime}(x-\ell)-\omega_{\lambda}^{2} \rho(x-\ell)\right) \quad \text { for } \quad \ell \leq x \leq \ell+1
$$

and $r_{\lambda}(x)=0$ for other $x$ from set $\{x:|x|>\ell\}$. In view of (39), we have the bound

$$
\left|r_{\lambda}(x)\right| \leq c_{1} \lambda^{4} \quad \text { for } \quad|x| \geq \ell
$$

because $\rho$ and $\rho^{\prime \prime}$ are bounded on $[0,1]$. Next, we calculate $r_{\lambda}$ for $|x|<\ell$. Recalling (4) and (23)-(25), we derive

$$
\begin{aligned}
r_{\lambda}= & \left(-\frac{d^{2}}{d x^{2}}+U+\lambda V_{\lambda}+\omega_{\lambda}^{2}\right) \psi_{\lambda} \\
= & \left(-\frac{d^{2}}{d x^{2}}+U+\lambda V+\lambda^{2} V_{1}+\lambda^{2} g_{\lambda}+\omega_{\lambda}^{2}\right)\left(u+\lambda v_{1}+\lambda^{2} v_{2}+\lambda^{3} v_{3}\right) \\
= & \left(-u^{\prime \prime}+U u\right)+\lambda\left(-v_{1}^{\prime \prime}+U v_{1}+V u\right)+\lambda^{2}\left(-v_{2}^{\prime \prime}+U v_{2}+V v_{1}\right. \\
& \left.+V_{1} u+\omega_{0}^{2} u+g_{\lambda} u\right)+\lambda^{3}\left(-v_{3}^{\prime \prime}+U v_{3}+f_{3, \lambda}\right)+\lambda^{4} R_{\lambda}=\lambda^{4} R_{\lambda},
\end{aligned}
$$

where the norm $\left\|R_{\lambda}\right\|_{L_{2}(\mathcal{I})}$ is bounded uniformly with respect to $\lambda$. From this we conclude that $\left\|r_{\lambda}\right\|_{L_{2}(\mathcal{I})}=O\left(\lambda^{4}\right)$, and hence that $\left\|r_{\lambda}\right\|=O\left(\lambda^{4}\right)$ as $\lambda \rightarrow 0$, in view of (41). Finally we have

$$
\left\|\left(H_{\lambda}+\omega_{\lambda}^{2} I\right) \phi_{\lambda}\right\|=\left\|\psi_{\lambda}\right\|^{-1}\left\|r_{\lambda}\right\| \leq c \lambda^{9 / 2}
$$

as $\lambda \rightarrow 0$, by Proposition 3 .

Owing to Lemmas 1 and 2 , the operator $H_{\lambda}$ possesses a negative eigenvalue $e_{\lambda}$ satisfying the bound $\left|e_{\lambda}+\lambda^{2}\left(\omega_{0}+\lambda \omega_{1, \lambda}+\lambda^{2} \omega_{2, \lambda}\right)^{2}\right| \leq c \lambda^{9 / 2}$. Since

$$
\left(\omega_{0}+\lambda \omega_{1, \lambda}+\lambda^{2} \omega_{2, \lambda}\right)^{2}-\left(\omega_{0}+\lambda \omega_{1}\right)^{2} \sim 2 \omega_{0} \lambda\left(\omega_{1, \lambda}-\omega_{1}\right)
$$

and $\omega_{1, \lambda}-\omega_{1}=o(1)$ as $\lambda \rightarrow 0$, we derive the asymptotic formula

$$
e_{\lambda}+\lambda^{2}\left(\omega_{0}+\lambda \omega_{1}\right)^{2}=o\left(\lambda^{3}\right),
$$

which we rewrite as $\left(\lambda^{-1} \sqrt{-e_{\lambda}}\right)^{2}-\left(\omega_{0}+\lambda \omega_{1}\right)^{2}=o(\lambda)$. From this we immediately deduce that $\lambda^{-1} \sqrt{-e_{\lambda}}+\omega_{0}+\lambda \omega_{1}=o(\lambda)$, and hence that

$$
\sqrt{-e_{\lambda}}=-\lambda\left(\omega_{0}+\lambda \omega_{1}+o(\lambda)\right)
$$

as $\lambda \rightarrow 0$, which completes the proof of Theorem 1 .

4.2. Proof of Theorem 2. Now we consider the critical case when inequality (5) turns into the equality

$$
\int_{\mathbb{R}} V u^{2} d x=0 .
$$

Hence $\omega_{0}=0$ in view of (4.1). Therefore $v_{0}=v_{*}$ and (38) becomes

$$
\omega_{1}=\frac{1}{\theta^{2}+1}\left(\int_{\mathbb{R}} V v_{*} u d x+\int_{\mathbb{R}} V_{1} u^{2} d x\right) .
$$

We must prove that a negative eigenvalue of $H_{\lambda}$ exists, the key point being that this existence assertion follows from inequality $\omega_{1}<0$. Indeed, almost eigenfunction $\psi_{\lambda}$ given by (22) belongs to $L_{2}(\mathbb{R})$ if $\omega_{\lambda}=\lambda^{2}\left(\omega_{1, \lambda}+\lambda \omega_{2, \lambda}\right)$ is negative, at least for small $\lambda$. Condition (9) ensures $\omega_{1, \lambda}<0$ and thereby $\omega_{\lambda}<0$ for $\lambda$ small enough.

By Proposition 3, we have $\left\|\psi_{\lambda}\right\| \sim a \lambda^{-1}$ as $\lambda \rightarrow 0$, provided $\omega_{0}=0$. Therefore estimate (42) can be improved $\left\|\left(H_{\lambda}+\omega_{\lambda}^{2}\right) \phi_{\lambda}\right\| \leq c \lambda^{5}$ and then

$$
\left|e_{\lambda}+\lambda^{4}\left(\omega_{1, \lambda}+\lambda \omega_{2, \lambda}\right)^{2}\right| \leq c_{1} \lambda^{5} .
$$


As in the proof of Theorem 1, if we rewrite this bound in the form

$$
\left(\lambda^{-2} \sqrt{-e_{\lambda}}\right)^{2}-\left(\omega_{1, \lambda}+\lambda \omega_{2, \lambda}\right)^{2}=O(\lambda),
$$

then we derive $\lambda^{-2} \sqrt{-e_{\lambda}}=-\omega_{1, \lambda}+O(\lambda)=-\omega_{1}+o(1)$. Finally, we have

$$
\sqrt{-e_{\lambda}}=-\lambda^{2}\left(\omega_{1}+o(1)\right) \quad \text { as } \quad \lambda \rightarrow 0
$$

and this is precisely the assertion of Theorem 2 .

4.3. Proof of Corollary 3. This statement differs from all earlier proved by the fact that here the threshold eigenvalue exists for both positive and negative $\lambda$ small enough. For the case $V_{\lambda}=V$ this result has been proved by Simon [1].

Since $\int_{\mathbb{R}} V d x=0$, from (16) we observe $\omega_{0}=0$ and

$$
\omega_{1}=\frac{1}{4} \iint_{\mathbb{R}^{2}} V(x)|x-y| V(y) d x d y .
$$

Proposition 4. If $V$ is a function of zero mean, then

$$
\iint_{\mathbb{R}^{2}} V(x)|x-y| V(y) d x d y=-2 \int_{\mathbb{R}}\left(\int_{-\infty}^{x} V(y) d y\right)^{2} d x .
$$

Proof. From $\int_{\mathbb{R}} V d x=0$ we immediately deduce

$$
\begin{aligned}
\int_{-\infty}^{x} V(y) d y & =-\int_{x}^{+\infty} V(y) d y \\
\int_{\mathbb{R}} V(x) \int_{-\infty}^{x} y V(y) d y d x & =-\int_{\mathbb{R}} V(x) \int_{x}^{+\infty} y V(y) d y d x .
\end{aligned}
$$

Therefore

$$
\begin{aligned}
\iint_{\mathbb{R}^{2}} V(x)|x-y| V(y) d x d y=\int_{\mathbb{R}} V(x) \int_{-\infty}^{x}(x-y) V(y) d y d x \\
+\int_{\mathbb{R}} V(x) \int_{x}^{+\infty}(y-x) V(y) d y d x=2 \int_{\mathbb{R}} x V(x) \int_{-\infty}^{x} V(y) d y d x \\
\quad-2 \int_{\mathbb{R}} V(x) \int_{-\infty}^{x} y V(y) d y d x=4 \int_{\mathbb{R}} x V(x) \int_{-\infty}^{x} V(y) d y d x
\end{aligned}
$$

because integrating by parts yields

$$
\int_{\mathbb{R}} V(x) \int_{-\infty}^{x} y V(y) d y d x=-\int_{\mathbb{R}} x V(x) \int_{-\infty}^{x} V(y) d y d x .
$$

The proof is completed by showing that

$$
\int_{\mathbb{R}} x V(x) \int_{-\infty}^{x} V(y) d y d x=-\frac{1}{2} \int_{\mathbb{R}}\left(\int_{-\infty}^{x} V(y) d y\right)^{2} d x .
$$

In view of this proposition, if potential $V$ is different from zero, then $\omega_{1}<0$. Hence $\omega_{\lambda}=\lambda^{2} \omega_{1, \lambda}+\lambda^{3} \omega_{2, \lambda}$ is negative for $\lambda$ small enough, positive or negative.

Acknowledgments. The author is indebted to the unknown Referee for a careful reading of this paper. The first version of the paper was significantly improved by the suggestions of the Referee. 


\section{REFERENCES}

1. B. Simon, The bound state of weakly coupled Schrödinger operators in one and two dimensions, Annals of Physics 97 (1976), no. 2, 279-288.

2. M. Klaus, On the bound state of Schrödinger operators in one dimension, Annals of Physics 108 (1977), no. 2, 288-300.

3. M. Klaus and B. Simon, Coupling constant thresholds in nonrelativistic quantum mechanics. I. Short-range two-body case, Annals of Physics 130 (1980), no. 2, 251-281.

4. M. Klaus and B. Simon, Coupling constant thresholds in nonrelativistic quantum mechanics, Comm. Math. Phys. 78 (1980), no. 2, 153-168.

5. B. Simon, On the absorption of eigenvalues by continuous spectrum in regular perturbation problems, J. Funct. Anal. 25 (1977), no. 4, 338-344.

6. M. Klaus, Some applications of the Birman-Schwinger principle, Helvetica Physica Acta 55 (1982), no. 1, 49-68.

7. Yu. Golovaty, On coupling constant thresholds in one dimension. arXiv:1905.10766 [math.SP] (2019); submitted to Letters in Mathematical Physics.

8. R. Blankenbecler, M. L. Goldberger, B. Simon, The bound states of weakly coupled long-range one-dimensional quantum Hamiltonians, Annals of Physics 108 (1977), no. 1, 69-78.

9. J. Rauch, Perturbation theory for eigenvalues and resonances of Schrödinger Hamiltonians, J. Funct. Anal. 35 (1980), no. 3, 304-315.

10. S. Albeverio, F. Gesztesy, and R. Høegh-Krohn, The low energy expansion in nonrelativistic scattering theory, Ann. Inst. H. Poincaré Sect. A (NS) 37 (1982), 1-28.

11. D. Bollé, F. Gesztesy, and S. F. J. Wilk, A complete treatment of low-energy scattering in one dimension, J. Operator Theory 13 (1985), no. 1, 3-31.

12. H. Holden, On coupling constant thresholds in two dimensions, J. Operator Theory, 14 (1985), no. 2, 263-276.

13. F. Gesztesy and H. Holden, A unified approach to eigenvalues and resonances of Schrödinger operators using Fredholm determinants, J. Math. Anal. Appl. 123 (1987), no. 1, 181-198.

14. A. Jensen, M. Melgaard, Perturbation of eigenvalues embedded at a threshold, Proceedings of the Royal Society of Edinburgh Section A: Mathematics, 132 (2002), no. 1, 163-179.

15. R. R. Gadyl'shin, Local perturbations of the Schrödinger operator on the axis, Theoretical and Mathematical Physics 132 (2002), no. 1, 976-982.

16. R. R. Gadyl'shin, Local perturbations of the Schrödinger operator on the plane, Teoreticheskaya i Matematicheskaya Fizika 138 (2004), no. 1, 41-54.

17. D. I. Borisov, R. R. Gadyl'shin, The spectrum of the Schrödinger operator with a rapidly oscillating compactly supported potential, Theoretical and Mathematical Physics 147 (2006), no. 1, 496-500.

18. S. Albeverio, L. Nizhnik, On the number of negative eigenvalues of a one-dimensional Schrödinger operator with point interactions, Lett. Math. Phys. 65 (2003), no. 1, 27-35.

19. F. Gesztesy, B. Simon, A short proof of Zheludev's theorem, Trans. Amer. Math. Soc. 335 (1993), no. 1, 329-340.

20. S. Fassari, M. Klaus, Coupling constant thresholds of perturbed periodic Hamiltonians, J. Math. Phys. 39 (1998), no. 9, 4369-4416.

21. G. Scharf and W. F. Wreszinski, Stability for the Korteweg-de Vries equation by inverse scattering theory, Annals of Physics 134 (1981), no. 1, 56-75.

22. M. I. Weinstein, Excitation thresholds for nonlinear localized modes on lattices, Nonlinearity 12 (1999), no. 3, 673.

23. P. G. Kevrekidis, K. Ø. Rasmussen, and A. R. Bishop, The discrete nonlinear Schrödinger equation: a survey of recent results, International Journal of Modern Physics B 15 (2001), no. 21, 2833-2900.

24. M. V. Fedoruyk, V. M. Babich, V. F. Lazutkin, ...\& B. R. Vainberg, Partial Differential Equations V: Asymptotic Methods for Partial Differential Equations, Encyclopaedia of Mathematical Sciences, vol. 5, Springer Science \& Business Media, 1999.

Department of Mechanics and Mathematics, Ivan Franko National University of Lviv, 1 UNIVERSYTETSKA STR., LVIV, 79000, UKRAINE

E-mail address: yuriy.golovaty@lnu.edu.ua

Received 05/09/2019; Revised 31/10/2019 